\title{
ON ARHANGEL'SKIǏ'S CLASS MOBI
}

\author{
H. R. BENNETT
}

1. Introduction. In [1], A. V. Arhangel'skir defines the class MOBI and asks whether each topological space in MOBI is either developable, pointwise paracompact ${ }^{1}$ or a $p$-space. ${ }^{2}$ It is further asserted that each topological space in MOBI has closed sets $G_{\delta}{ }^{3}$ and asked if a Lindelöf space or a paracompact space in MOBI is metrizable. These questions are answered negatively.

The notation will follow [4] and all spaces will be at least Hausdorff.

\section{The class MOBI.}

Definition 2.1. The class MOBI is the intersection of all classes of topological spaces satisfying the two conditions:

(i) Every metric space belongs to the class.

(ii) The image of a space in the class under a compact open map ${ }^{4}$ is also in the class.

TheOREM 2.2. A topological space $y$ is in MOBI if and only if there is a metric space $M$ and a finite set $\left\{\phi_{1}, \cdots, \phi_{n}\right\}$ of compact open maps such that $\left(\phi_{n} \circ \cdots \circ \phi_{1}\right)(M)=Y$.

Proof. Let MOBI $=\bigcap\left\{C_{\alpha} \mid \alpha \in A\right\}$ and let $B=\{X \mid X$ is a metric space $\} \cup\left\{Y \mid\right.$ there is a metric space $M$ and a finite set $\left\{\phi_{1}, \cdots, \phi_{n}\right\}$ of compact open maps such that $\left.\left(\phi_{n} \circ \cdots \circ \phi_{1}\right)(M)=Y\right\}$.

There is an $\alpha$ in $A$ such that $B=C_{\alpha}$ since if $Y$ is in $B$, then either $Y$ is a metric space and, consequently, in $C_{\alpha}$ or $Y=\left(\phi_{n} \circ \cdots \circ \phi_{1}\right)(M)$ where $M$ is metric and each $\phi_{i}, 1 \leqq i \leqq n$, are compact open maps. Thus if $\phi$ is a compact open map $\phi(Y)=\phi\left(\left(\phi_{n} \circ \cdots \circ \phi_{1}\right)(M)\right)$ is, by definition, in $B$. Thus $B=C_{\alpha} \supseteq$ MOBI.

Received by the editors June 9, 1969.

AMS 1969 subject classifications. Primary 5460; Secondary 5438, 5450.

${ }^{1}$ A space is pointwise paracompact (=weakly paracompact in Russian) if each open cover has a point finite open refinement.

${ }^{2}$ A topological space $S$ is a $p$-space provided that for some Hausdorff compactification $T$ of $S$ there is a sequence $P_{1}, P_{2}, \cdots$ of collections of open subsets of $T$, each of which covers $S$, such that if $x$ is any element of $S$, then $\bigcap_{i=1}^{\infty}\left\{U\left\{p \in P_{i} \mid x \in p\right\}\right\}$ is contained in $S$.

${ }^{3}$ A set $A$ is a $G_{\delta}$ if it is the countable intersection of open sets.

- A map is a continuous function. A map is compact open if the image of an open set is open and if the inverse image of a point is compact. 
For each $\alpha$ in $A, B \subseteq C_{\alpha}$. For if $Y$ is in $B$, then either $Y$ is metric and in $C_{\alpha}$ or $Y=\left(\phi_{n} \circ \cdots \circ \phi_{1}\right)(M)$ where $M$ is metric and the $\phi_{i}$, $1 \leqq i \leqq n$, are compact open maps. Since $M$ is in $C_{\alpha}, \phi_{1}(M)$ is also in $C_{\alpha}$ and inductively $\left(\phi_{n} \circ \cdots \circ \phi_{1}\right)(M)=Y$ is in $C_{\alpha}$. Thus $B \subseteq C_{\alpha}$ for each $\alpha$. It follows that $B=\bigcap\left\{C_{\alpha} \mid \alpha \in A\right\}=$ MOBI.

COROLlaRy 2.3. Each pointwise paracompact, developable Hausdorff space is in MOBI.

Proof. Theorem 2.2 and Theorem 5 of [5].

Definition 2.4. A sequence $G_{1}, G_{2}, \cdots$ of collections of open subsets of a topological space $X$ is called a quasi-development for $X$ provided that for each point $p$ of $X$ and each open set $R$ containing $p$ there is a natural number $n$ such that $p$ belongs to some element of $G_{n}$ and each element of $G_{n}$ that contains $p$ lies in $R$. If, in addition, each $G_{n}$ is a cover of $X$, then $G_{1}, G_{2}, \cdots$ is a development. A space having a (quasi-) development (see [3]) is said to be (quasi-) developable.

THEOREM 2.5. There exists a regular, Lindelöf, hereditarily paracompact, quasi-developable space $Y^{5}$ in MOBI that is neither developable, a p-space, nor has closed sets $G_{\delta}$.

Proof. Let $Q$ be an uncountable subset of $[0,1]$ whose only compact subsets are countable; such spaces exist [7, p. 422]. Let $T=[0,1]-Q$ and if $N$ denotes the natural numbers let $X$ $=\{(x, y) \mid 0 \leqq x \leqq 1, y=0$ or $x \in Q, y \in N\}$. Topologize $X$ by letting a set be open if it is the union of any of the following types of sets:

(i) $\{(x, y)\}$ if $0 \leqq x \leqq 1$ and $y>0$,

(ii) $\{(x, 0)\} \cup\{(x, y) \mid y>c\}$ if $x \in Q$ and $c>0$,

(iii) $\{(x, 0) \mid x \in T, \quad a<x<b\} \cup\{(x, y) \mid a<x<b, \quad x \in Q, \quad y>c\}$ where $a<b$ and $c>0$.

It follows that $X$ is a Hausdorff, pointwise paracompact, developable space. Therefore, by Corollary $2.3, X$ is a MOBI.

Let $Y$ be $[0,1]$ retopologized to make the points of $Q$ discrete. As noted in [6], $Y$ is hereditarily paracompact and Lindelöf. Clearly $Y-Q$ is closed and not a $G_{\delta}$ in $Y$; thus $Y$ is not developable. If $\left\{B_{1}, B_{2}, \cdots\right\}$ is a countable base for $[0,1]$, by letting $G_{1}$ $=\{\{x\} \mid x \in Q\}$ and, for $i>1, G_{i}=\left\{B_{i-1}\right\}$, it follows that $G_{1}, G_{2}, \ldots$ is a quasi-development for $Y$. It is shown in [6] that the product of $Y$ with a metric space (the irrationals) is not normal. Thus, by a result of A. V. Arhangel'skii (Corollary 1 of Theorem 16 in [2]) it follows that $Y$ is not a $p$-space.

5 The space in Example 2 of [8] is similar to the space $Y$. 
Let a map $\phi$ be defined by $\phi((x, y))=x$. It follows that $\phi$ is an open compact map with domain $X$ and range $Y$. By Theorem 2.2 $Y$ is in MOBI.

Thus most of the questions in the group of problems 5.7 in [1] are answered negatively. Example 3 of [8] shows that each element of MOBI need not be pointwise paracompact.

QUESTION 1. Is each member of MOBI a quasi-developable space? Question 2.6 If the members of MOBI are restricted to completely regular spaces, can the questions in 5.7 of [1] still be answered negatively?

\section{BIBLIOGRAPHY}

1. A. V. Arhangel'skil, Mappings and spaces, Uspehi Mat. Nauk 21 (1966), no. 4 (130), 133-184 = Russian Math. Surveys 21 (1966), no. 4, 115-162. MR 37 \#3534.

2. - On a class of spaces containing all metric and all locally compact spaces, Dokl. Akad. Nauk SSSR 151 (1963), 751-754=Soviet Math. Dokl. 4 (1963), 10511055. MR 27 \#2959.

3. H. R. Bennett, Quasi-developable spaces, Dissertation, Arizona State University, Tempe, Arizona, 1968.

4. J. Dugundi, Topology, Allyn and Bacon, Boston, Mass., 1966. MR 33 \#1824.

5. Sitiro Hanai, On open mappings. II, Proc. Japan Acad. 37 (1961), 233-238. MR 23 \#A4116.

6. E. Michael, The product of a normal space and a metric space need not be normal, Bull. Amer. Math. Soc. 69 (1963), 375-376. MR 27 \#2956.

7. C. Kuratowski, Topologïe. Vol. I, PWN, Warsaw, 1958; English transl., Academic Press, New York and PWN, Warsaw, 1966. MR 19, 873; MR 36 \#839; 840.

8. H. H. Wicke and J. M. Worrell, Open continuous mappings of spaces having bases of countable order, Duke Math. J. 34 (1967), 255-271. MR 35 \#979.

Texas Technological University, Lubbock, Texas 79406

${ }^{6}$ This question is due to the referee, whom the author would like to thank for his valuable suggestions. 